\title{
Exploiting lymphatic transport and complement activation in nanoparticle vaccines
}

\author{
Sai T Reddy ${ }^{1}$, André J van der Vlies ${ }^{1}$, Eleonora Simeoni ${ }^{1}$, Veronique Angeli ${ }^{2,4}$, Gwendalyn J Randolph ${ }^{2}$, \\ Conlin P O’Neil ${ }^{1}$, Leslie K Lee ${ }^{1}$, Melody A Swartz ${ }^{1,3}$ \& Jeffrey A Hubbell ${ }^{1,3}$
}

Antigen targeting ${ }^{1-5}$ and adjuvancy schemes ${ }^{6,7}$ that respectively facilitate delivery of antigen to dendritic cells and elicit their activation have been explored in vaccine development. Here we investigate whether nanoparticles can be used as a vaccine platform by targeting lymph noderesiding dendritic cells via interstitial flow and activating these cells by in situ complement activation. After intradermal injection, interstitial flow transported ultra-small nanoparticles $(25 \mathrm{~nm})$ highly efficiently into lymphatic capillaries and their draining lymph nodes, targeting half of the lymph noderesiding dendritic cells, whereas $100-n m$ nanoparticles were only $10 \%$ as efficient. The surface chemistry of these nanoparticles activated the complement cascade, generating a danger signal in situ and potently activating dendritic cells. Using nanoparticles conjugated to the model antigen ovalbumin, we demonstrate generation of humoral and cellular immunity in mice in a size- and complementdependent manner.

Technologies for presentation of protein or peptide antigens as vaccines must address two fundamental issues: efficient delivery of antigen to dendritic cells and subsequent dendritic cell activation to trigger adaptive immunity. Strategies for antigen delivery have recently focused on in vivo targeting of dendritic cells through the use of monoclonal antibodies either fused with protein ${ }^{1,2,4}$ or grafted to the surface of microparticles ${ }^{3}$. Such strategies often target peripheral dendritic cells in skin. Antigen delivery to lymph nodes might provide an attractive alternative, because dendritic cells are present in much higher concentration in these lymph nodes. To initiate adaptive immunity, adjuvants must induce dendritic cell maturation. Dendritic cells are often matured by 'danger signals' that work through pathways of innate immunity such as activation of Toll-like receptors (TLRs) and inflammatory cytokine receptors. Whereas experimental vaccines using danger signals have shown promise $e^{6,7}$, risk of toxicity, physiological transport limitations and economic feasibility remain as potentially limiting issues.

As a nanoparticulate platform for antigen delivery, we used Pluronic-stabilized polypropylene sulfide (PPS) nanoparticles ${ }^{8}$.
These nanoparticles have a hydrophobic core of crosslinked PPS, which degrades by becoming water soluble under oxidative conditions
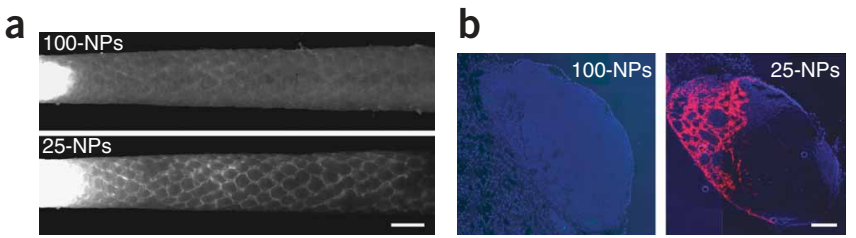

C

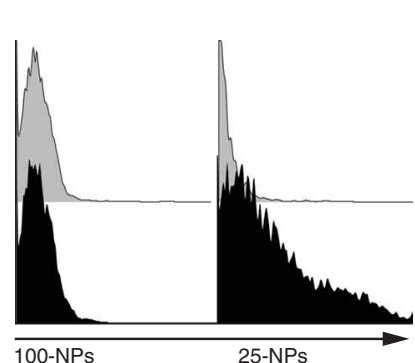

d

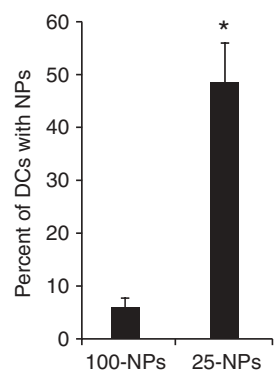

Figure 1 Ultra-small nanoparticles accumulate in lymph nodes after intradermal injection, whereas slightly larger ones do not. (a) Fluorescence microlymphangiography is convenient for imaging lymphatic uptake because the lymphatic capillaries all drain in one direction, toward the base of the tail. Shown is a co-infusion into mouse-tail skin with 100- and 25-nm fluorescently labeled nanoparticles (100- and 25-NPs, respectively) where the 25-NPs enter the dermal lymphatic capillary network much more efficiently than 100-NPs do. Scale bar, $1 \mathrm{~mm}$. (b) The $25-\mathrm{nm}$, but not the 100-nm, nanoparticles are visible in mouse lymph node sections $24 \mathrm{~h}$ after injection. Cell nuclei shown in blue (DAPI); scale bar, $200 \mu \mathrm{m}$. Images in a and $\mathbf{b}$ are representative of at least three independent experiments. (c) Flow cytometry histograms show $\mathrm{CD} 11 \mathrm{c}^{+}$dendritic cells isolated from the draining lymph nodes after intradermal co-injection of fluorescently labeled 100- and 25-NPs (black) or phosphate-buffered saline (PBS) control (gray).

Results are representative of at least three independent experiments. (d) Calculations of the fraction of dendritic cells that have internalized NPs after a co-injection as in c supports the hypothesis that lymph node dendritic cells are effectively targeted after intradermal injection of smaller but not larger nanoparticles. Values are the means from six independent experiments; error bars correspond to s.d. ${ }^{*}, P<0.001$.

\footnotetext{
${ }^{1}$ Institute of Bioengineering, École Polytechnique Fédérale de Lausanne (EPFL), CH-1015 Lausanne, Switzerland. ${ }^{2}$ Department of Gene and Cell Medicine, Mount Sinai School of Medicine, New York, New York, 10029 USA. ${ }^{3}$ Institute of Chemical Sciences and Engineering, École Polytechnique Fédérale de Lausanne (EPFL), CH-1015 Lausanne, Switzerland. ${ }^{4}$ Present address: Department of Microbiology, Immunology Programme, National University of Singapore, Singapore 117456. Correspondence
} and request for materials should be addressed to M.A.S (melody.swartz@epfl.ch) or J.A.H (jeffrey.hubbell@epfl.ch). 
a

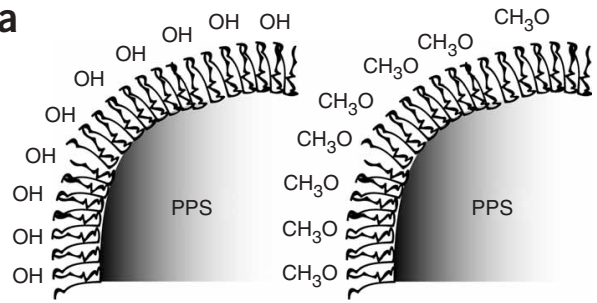

C
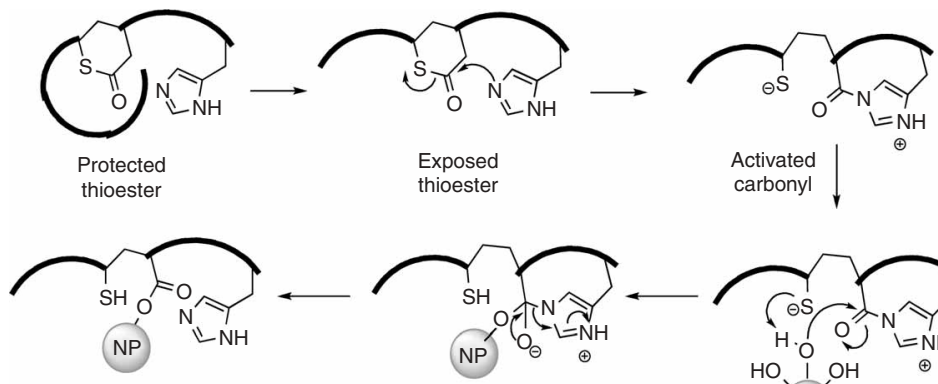

Covalent bond between $\mathrm{OH}$ and $\mathrm{C} 3 \mathrm{~b}$
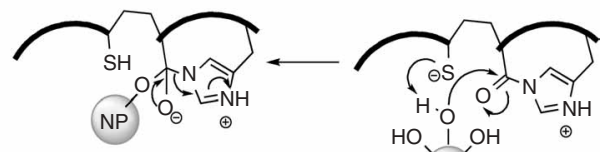

$\mathrm{HO}_{2} \mathrm{I} \mathrm{OH}$

$\mathrm{HO}-\mathrm{NP}-\mathrm{OH} \quad$ Attack by $\mathrm{HO}_{\mathrm{OH}} \mathrm{OH} \quad \mathrm{OH}$ group

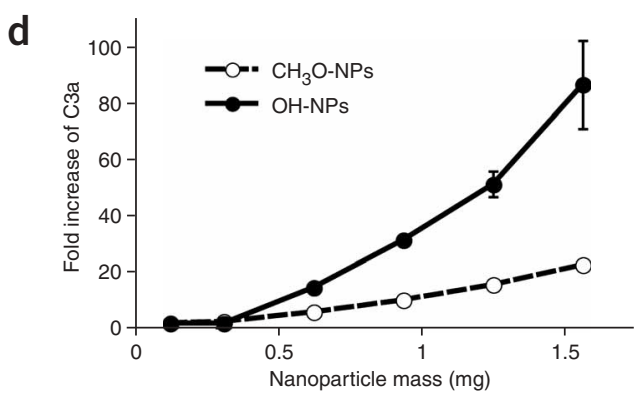

Figure 2 Polyhydroxylated nanoparticle surfaces activate complement. (a) Synthesis and stabilization with two different forms of Pluronic allowed the generation of polyhydroxylated- or polymethoxylatednanoparticles. (b) The $\alpha, \omega$-terminal $\mathrm{OH}$ groups on Pluronic could be converted to $\mathrm{OCH}_{3}$ groups. (c) The proposed mechanism where $\mathrm{OH}$ groups on the polyhydroxylated nanoparticles can bind to the exposed thioester of C3b to activate complement by the alternative pathway. (d) Nanoparticleinduced complement activation, as measured through C3a presence in human serum after incubation with nanoparticles, was demonstrated to be high with polyhydroxylated nanoparticles but low with polymethoxylated nanoparticles $\left(\mathrm{OH}-\right.$ and $\mathrm{CH}_{3} \mathrm{O}-\mathrm{NPs}$, respectively). Results are normalized to control of serum incubation with PBS. Values are means of three independent experiments; error bars correspond to standard error of mean, s.e.m.

(such as those found within the lysosome), and a hydrophilic surface corona of Pluronic, a block copolymer of polyethylene glycol and polypropylene glycol ${ }^{8}$. The Pluronic enables good size control down to $\sim 20 \mathrm{~nm}$ during inverse emulsion polymerization ${ }^{8}$. By selecting the lymph node as our target, we seek to achieve localization of the nanoparticles where dendritic cells exist in high concentrations and where a substantial fraction of dendritic cells are phenotypically and functionally immature ${ }^{9}$ and thus able to process new antigen. This is in contrast to the common approach of targeting dendritic cells in peripheral tissues like skin or muscle ${ }^{10}$, where dendritic cells reside in much lower numbers and must travel to the lymph node after antigen uptake ${ }^{11}$, permitting premature antigen presentation ${ }^{12}$. To compensate for low dendritic cell density in peripheral tissues, many investigators have explored biomolecular approaches to dendritic cell targeting. Whereas particles can be taken up nonspecifically by peripheral dendritic cells, as demonstrated with polymer microparticles containing antigen-encoding $\mathrm{DNA}^{5,13}$, viruslike particles ${ }^{14}$ or polystyrene nanobeads ${ }^{15}$, investigators have also conjugated monoclonal antibodies directed to dendritic cellsurface receptors such as DEC205 or 33D1 to protein antigen ${ }^{1,2}$ or to the surface of liposomes or microparticles ${ }^{3,16}$ for more specific targeting. However, such sophisticated cell-specific targeting may not be necessary if antigen could be delivered to the lymph node, where dendritic cells are present in high numbers.

To target dendritic cells in the lymph node, we exploited a biophysical mechanisminterstitial flow-to access the lymphatic system as a low-resistance delivery route that leads to lymph nodes. The lymphatic system constantly drains fluid and macromolecules from the interstitial space, creating small interstitial flows on the order of $0.1-1 \mu \mathrm{m} / \mathrm{s}^{17,18}$. We take advantage of this basic physiological phenomenon by using nanoparticles that are so small that they are convected by this interstitial flow through the interstitial matrix into the draining lymphatic capillary bed. In previous work, size was shown to be the most critical factor affecting lymphatic uptake of particle-based delivery vehicles ${ }^{19}$, probably based on the network structure of the interstitial extracellular matrix and the valve-like overlapping lymphatic cell-cell junctions, through which entry of particles depends on extracellular matrix hydration ${ }^{17}$. Larger nanoparticles have also been explored by others ${ }^{20}$, but these enter the lymphatics much less efficiently than ultra-small nanoparticles and therefore are likely to target peripheral more than lymph node-resident dendritic cells.

To explore lymphatic uptake, we performed fluorescence microlymphangiography ${ }^{21}$ by constant pressure infusion of nanoparticles into the tip of mouse tail skin; $25-\mathrm{nm}$ but not $100-\mathrm{nm}$ nanoparticles were quickly and efficiently taken up by lymphatic vessels (Fig. 1a). Once within the lymphatic vasculature, the 25-nm nanoparticles were efficiently transported to the draining lymph node (Fig. 1b) and could be retained there for at least $120 \mathrm{~h}$ after injection, were colocalized exclusively with macrophages and dendritic cells in the subcapsular sinus, and were not found within $\mathrm{T}$ - or B-cell zones ${ }^{19}$. The $25-\mathrm{nm}$ nanoparticles were found within $\sim 50 \%$ of dendritic cells isolated from the lymph node (Fig. 1c,d), whereas $100-\mathrm{nm}$ nanoparticles were found within only $6 \%$ of dendritic cells and could not be visualized within the draining lymph node after $24 \mathrm{~h}$ (Fig. 1b-d). It is likely that these larger nanoparticles entered the lymph node after internalization by peripheral dendritic cells and migration to the node, because dendritic cells take up large particles in this size range as efficiently as smaller particles ${ }^{5,19}$. Thus, in addition to targeting peripheral dendritic cells in skin, nanoparticles that are small enough to readily access the lymphatic system from the interstitium can effectively target lymph node-resident dendritic cells. 


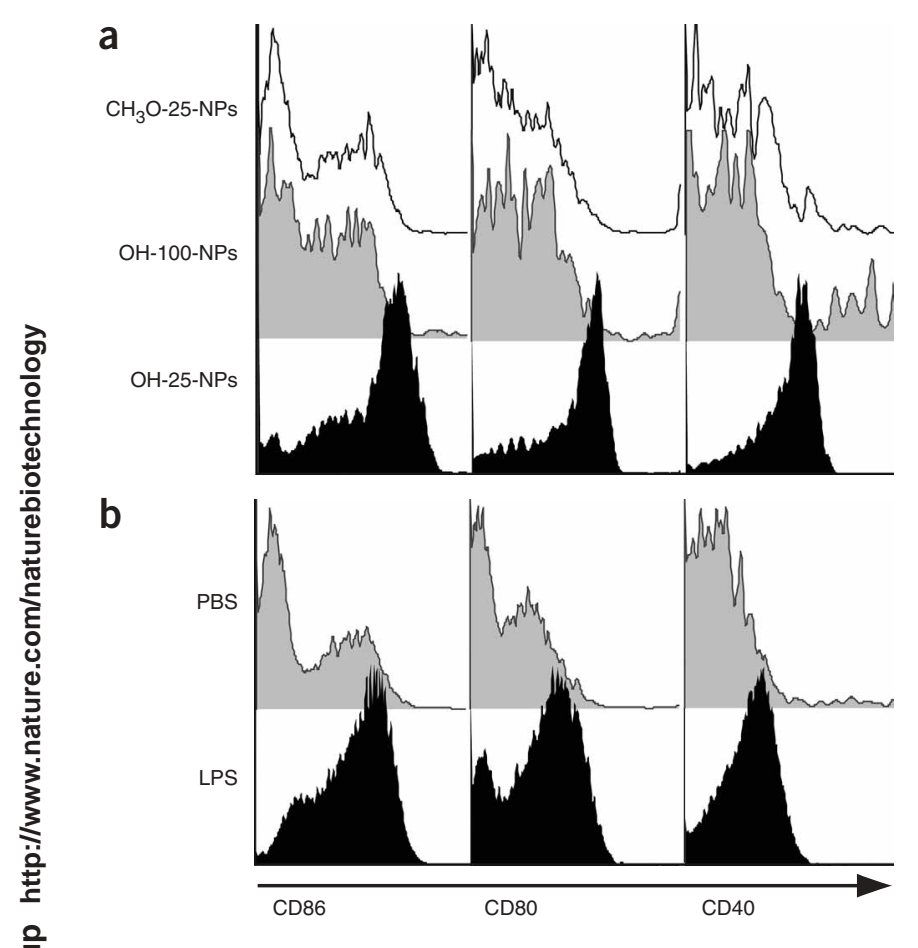

Next, we designed the nanoparticles to function themselves as an adjuvant, which is necessary to induce dendritic cell maturation and initiate adaptive immunity. Adjuvants are typically danger signals that work by activation of TLRs and inflammatory cytokine receptors; such adjuvants have been widely explored in experimental vaccine formulations $^{6,7}$. For example, molecular danger signals that mimic the effect of the endotoxin lipopolysaccharide (LPS), such as monophosphoryl lipid A, have been explored ${ }^{7}$, as well as the cytokines CD40 ligand and interferon (IFN)- $\gamma^{1,2,16}$. Delivery of danger signals with antigen by co-encapsulation in liposomes ${ }^{3,16}$, packaging into virus-like particles $^{14}$ or co-injection with dendritic cell-targeting antibody fusion proteins ${ }^{1,4,22}$ have been successful at producing adaptive immune responses, but despite such promising results, many complexities remain including toxicity, physiological transport and economic feasibility. As an alternative adjuvant strategy, we explored the possibility of using the complement cascade as a danger signal of innate immunity, designing our nanoparticles with a surface chemistry that spontaneously induces complement activation in situ.

Because the use of adjuvants based on known TLR activators has dominated vaccine research in recent years ${ }^{7}$, the complement system, another pathway of innate immunity, has been relatively unexplored as a danger signal adjuvant. Complement primarily serves as a biochemical defense system that clears pathogens nonspecifically, but it can also play a role in promoting antigen-specific immune responses ${ }^{23}$. Its ability to enhance humoral immunity has been exploited with molecular targeting adjuvants that enhance antigen delivery directly to $\mathrm{B}$ cells ${ }^{24}$. Only recently has it been discovered that complement can promote T-cell immunity ${ }^{25,26}$, but the molecular mechanisms and interactions with dendritic cells remain largely unidentified.

In the alternative pathway of the complement cascade, C3 is activated by spontaneous proteolytic cleavage to form the $\mathrm{C} 3 \mathrm{a}$ and C3b fragments. C3b undergoes a conformational change and exposes a thioester, which forms a reactive acyl-imidazole bond with a proximate histidine residue. The formed thiolate acts as a base and
Figure 3 Lymph node-targeting, complement-activating nanoparticles induce dendritic cell maturation in vivo. (a) Flow cytometry histograms showing $\mathrm{CD} 11 \mathrm{c}^{+}$dendritic cells isolated from mouse lymph nodes at $24 \mathrm{~h}$ after intradermal injection with either 25-nm polymethoxylated nanoparticles ( $\left.\mathrm{CH}_{3} \mathrm{O}-25-\mathrm{NPs}\right), 100-\mathrm{nm}$ polyhydroxylated-NPs $(\mathrm{OH}-100-\mathrm{NPs})$, or 25-nm polyhydroxylated-NPs (OH-25-NPs) demonstrate selective activation: $\mathrm{OH}-25-\mathrm{NPs}$, which both target lymph nodes and strongly activate complement, induce upregulation of the dendritic cell maturation markers CD86, CD80 and CD40. (b) Histograms are also shown from mice that received injections of negative and positive controls, PBS and LPS, respectively; demonstrating that activation with $\mathrm{OH}-25-\mathrm{NPs}$ is comparable to activation with LPS. All results are representative of at least three independent experiments.

catalyzes the transfer of the acyl group to hydroxyl groups, prototypically located on carbohydrate residues that coat pathogens ${ }^{27}$. This mechanism also occurs with some nonnatural hydroxlyated material surfaces ${ }^{28,29}$, including Pluronic-coated materials ${ }^{30}$. Biomaterials scientists have typically sought to avoid complement activation in order to minimize effects such as implant rejection and clearance of systemic drug delivery vehicles. Here, we instead attempted to do the opposite and specifically designed a biomaterial that strongly activates complement so that it could generate a molecular adjuvant danger signal in situ. Indeed, we saw that nanoparticles stabilized by Pluronic (Fig. 2a) activated complement through Pluronic's $\alpha$ and $\omega$ terminal hydroxyl groups (Fig. $2 \mathbf{b}-\mathbf{d}$ ). To explore whether complement was activated exclusively by surface hydroxyls, we synthesized nanoparticles with $\alpha, \omega$-methoxy-Pluronic (Fig. 2b). These activated complement to a much reduced, but still nonzero extent (Fig. 2d), demonstrating that although the surface hydroxyls are critical for very high levels of complement activation, they are not the only feature on the nanoparticle surface involved in complement activation.

With the ability to target lymph node-resident dendritic cells with ultra-small nanoparticles and activate complement with their polyhydroxylated surfaces, we then determined that these properties could be combined to elicit dendritic cell maturation. Following intradermal injection into mouse dorsal foot skin of 25-nm polyhydroxylatednanoparticles, dendritic cells isolated $24 \mathrm{~h}$ after injection from the draining lymph nodes displayed a shift from the immature to the mature phenotype through upregulation of the co-stimulatory molecules CD86, CD80 and CD40 (Fig. 3a). Both lymph node-targeting and complement activation were necessary to elicit such an effect, because $100-\mathrm{nm}$ polyhydroxylated and 25-nm polymethoxylated nanoparticles did not induce dendritic cell maturation above control levels (Fig. 3a). Moreover, the dendritic cell maturation level induced by $25-\mathrm{nm}$ polyhydroxylated nanoparticles was very similar to that induced by the endotoxin LPS, a potent activator of TLR4 (Fig. 3b), further demonstrating that complement activation can be a very effective intrinsic danger signal for dendritic cells. Complement activation did not appear to significantly affect lymph node dendritic cell uptake as 25-nm polymethoxylated and polyhydroxylated nanoparticles were internalized by dendritic cells at similar levels (Supplementary Fig. 1 online). As a comparison we also injected commercially available 20 -nm polystyrene nanospheres (Invitrogen) with carboxylated surfaces. The polystyrene nanospheres accumulated in lymph nodes at similar levels as 25-nm polyhydroxylated nanoparticles but did not induce dendritic cell maturation (Supplementary Fig. 2 online). Thus, nanoparticles engineered to both target lymph nodes and strongly activate complement are potent maturation stimuli for dendritic cells.

To test the extent to which the lymph node-targeting, complementactivating nanoparticles induced an antigen-specific immune 
response, we conjugated ovalbumin, a model protein antigen, to nanoparticle surfaces (Supplementary Fig. 3 online). Ovalbumin conjugation did not significantly affect the nanoparticle size and the antigen remained coupled to the particle after delivery to the lymph node (Supplementary Fig. 4 online). Furthermore, the $25-\mathrm{nm}$ ovalbumin nanoparticles induced lymph node dendritic cell maturation at the same levels as unconjugated ovalbumin co-injected with LPS

(Fig. 4a). To measure T-cell proliferation, we used an adoptive transfer

experiment with OT-II mice (a transgenic mouse strain in which CD4 a

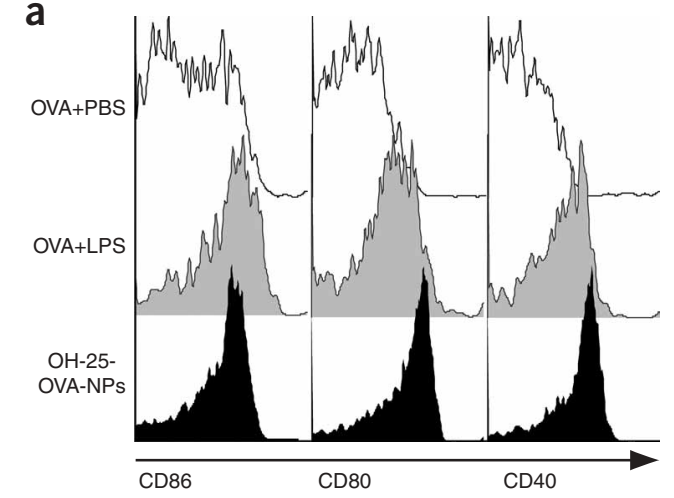

b

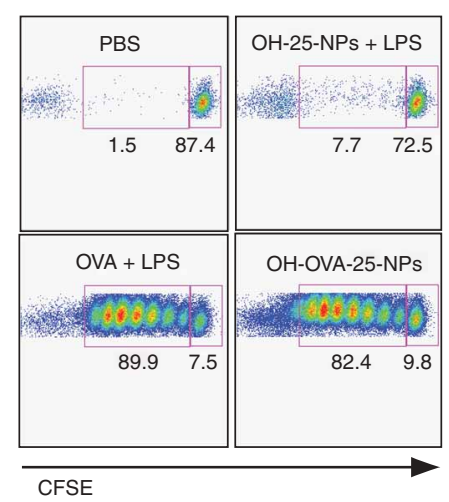

C

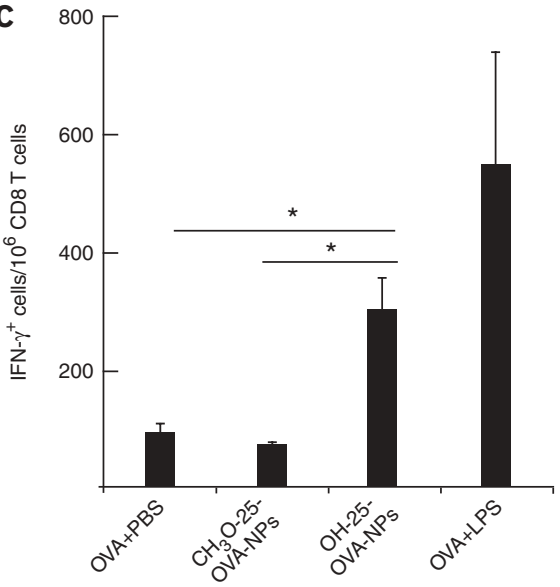

d

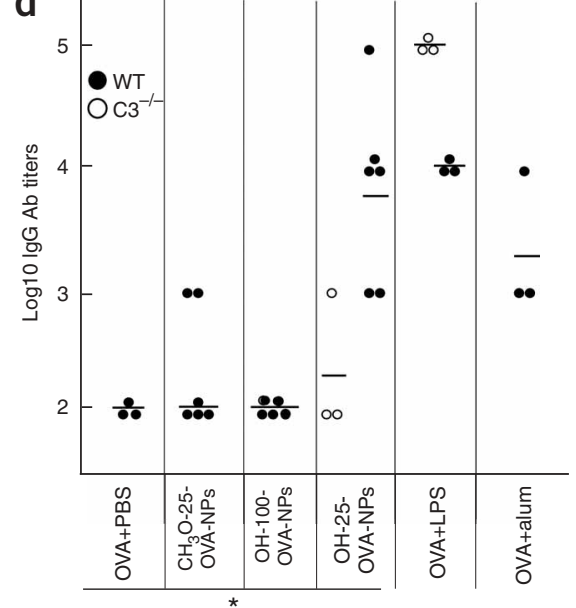

Figure 4 Lymph node-targeting, complement-activating nanoparticles induce antigen-specific adaptive immune responses. (a) Histograms display lymph node $\mathrm{CD} 11 \mathrm{c}^{+}$dendritic cell maturation profiles after intradermal injections into mice with either ovalbumin with PBS or LPS (+PBS or +LPS, respectively) or ovalbumin conjugated to 25 -nm polyhydroxylated nanoparticles (OH-25-ovalbumin-NPs). Dendritic cell activation is apparent for the $\mathrm{OH}-25$-ovalbumin nanoparticles, comparable to that obtained with ovalbumin+LPS. (b) Flow cytometry profiles show that lymph node $\mathrm{CD} 4{ }^{+} \mathrm{CD} 45.2^{+} \mathrm{T}$-cell proliferation after treatment with $\mathrm{OH}-25$-ovalbumin-NPs is comparable to that after treatment with ovalbumin+LPS as measured by dilution of 5-(6)-carboxyfluorescein diacetate succinimidyl diester (CFSE) labeled OT-II T cells. Results from $\mathbf{a}$ and $\mathbf{b}$ are representative of two to four independent experiments. (c) A restimulation assay was used to determine CD8 T-cell memory by IFN- $\gamma$ production. Mice received various intradermal injections of antigen and nanoparticle formulations. The graph shows CD8 T-cell memory after treatment with $\mathrm{OH}-25$-ovalbumin-NPs but not $\mathrm{CH}_{3} \mathrm{O}-25$-ovalbumin-NPs. Results are mean values from three mice in each group; error bars correspond to s.e.m. ${ }^{*}, P<0.05$. (d) Mice received single injections of different antigen and nanoparticle formulations, and serum was collected $21 \mathrm{~d}$ after injection. The graph shows antibody titers for anti-ovalbumin IgG, where each point represents an individual mouse and the bar represents the group mean. A strong humoral immune response was observed only for $\mathrm{OH}-25$-ovalbumin-NPs, but not larger or low-complement activating nanoparticles. When the $\mathrm{OH}-25$-ovalbumin-NPs were injected into $\mathrm{C} 3^{-/-}$mice (open circles), this response was abrogated (filled circles, wild type, WT), confirming the role of in situ complement activation as a danger signal. ${ }^{*}, P<0.05$.
$\mathrm{T}$ cells upregulate their ovalbumin-specific $\mathrm{T}$-cell receptors). Consistent with the results on dendritic cell activation, the 25-nm polyhydroxylated-ovalbumin-nanoparticles showed nearly the same capacity to induce T-cell proliferation as the positive control of ovalbumin co-injected with LPS, each producing approximately even daughter generations (Fig. 4b). Helper CD4 T cells play an cytotoxic CD8 $\mathrm{T}$ cells and in humoral immunity by stimulating antibodies. Therefore nanoparticle induction of strong antigen-specific CD4 T-cell proliferation provided the impetus to determine if cellular and humoral immunity could be induced.

Cellular immunity is critical for vaccines capable of inducing resistance to intracellular pathogens, and this is typically defined by the induction of cytotoxic T-cell memory. This is often measured by determining if CD8 T cells from immunized mice become activated, producing the inflammatory cytokine IFN- $\gamma$ after reexposure to an antigenic stimulus. Adjuvants that produce sufficient CD8 T-cell activation have thus far been elusive, however, an LPS synthetic derivative, monophosphoryl lipid A has shown potential ${ }^{7}$. Thus, we measured the ability of ultra-small, ovalbuminconjugated, polyhydroxylated nanoparticles to induce cellular immunity compared to LPS. We found that 25-nm polyhydroxylatedovalbumin-nanoparticles produced activated $\mathrm{T}$ cells at similar levels to unconjugated ovalbumin co-injected with LPS, and at significantly higher levels than 25-nm polymethoxylated-ovalbumin-nanoparticles (Fig. 4c). This demonstrated that downstream antigenspecific cellular immunity was attained with ovalbumin nanoparticles, but only when the complement-activating surface was present.

Another critical feature of vaccines is the ability to induce humoral immunity, the generation of a resistant level of circulating antibodies against a pathogenic antigen. To determine whether the nanoparticles could induce humoral immunity, we measured antibody titers in serum $21 \mathrm{~d}$ after a single injection. We found that $25-\mathrm{nm}$ polyhydroxylated-ovalbumin-nanoparticles produced strong levels of anti-ovalbumin IgG similar to injections of ovalbumin with LPS or the clinically used adjuvant alum. Significantly lower antibody titers were observed with 100-nm polyhydroxylatedovalbumin-nanoparticles and $25-\mathrm{nm}$ polymethoxylated-ovalbumin-nanoparticles

(Fig. 4d). As further confirmation of the importance of complement activation, we found that $\mathrm{C} 3^{-1-}$ mice produced substantially lower antibody titers when injected with 25-nm polyhydroxylated-ovalbuminnanoparticles than did wild-type controls (Fig. 4d), where injections of ovalbumin 
with LPS resulted in a normal antibody response in $\mathrm{C}^{-1-}$ mice. Thus, ovalbumin nanoparticles could only induce humoral immunity in the presence of both lymph node-targeting and complement activation.

Current vaccine technology research is dominated by strategies targeting peripheral dendritic cells, for example, in the skin or muscle, using molecular principles for dendritic cell targeting. Lymph noderesident dendritic cells are rarely considered as targets. Thus far, research with particulate systems in a size range small enough for lymphatic uptake such as virus-like particles ${ }^{14}$ and polystyrene nanobeads ${ }^{15}$ has not exploited lymphatic transport. Lymphatic delivery is often even prevented by aggregating antigen with alum to produce a depot effect. Adjuvant research is dominated by formulations using TLR activators and inflammatory cytokines as molecular danger signals $^{6,7}$, complement activation having been relatively unexplored. Our results highlight two alternative strategies for vaccination: interstitial-to-lymphatic flow to deliver antigen and adjuvant to lymph node-resident dendritic cells, and in situ complement activation to mature these cells. Whereas we present the nanoparticle system here as an implementation to explore these concepts, we do note its ease of fabrication and antigen conjugation, high stability and attractive affordability. Nevertheless, questions regarding toxicity, elimination and molecular interaction between complement and dendritic cells remain to be addressed to demonstrate the reported system as more than an implementation to explore these two strategies.

\section{METHODS}

Animals. BALB/c, C57BL6 and $\mathrm{C}^{-1-}$ (B6.129S4-C3tm1Crr/J), OT-II Tg (CD45.2) and CD45.1 mice (Jackson Immunoresearch) at 6-10 weeks old and weighing $20-30 \mathrm{~g}$, were used for this study. All protocols were approved by the Veterinary Authorities of the Canton Vaud according to Swiss law (protocol number nos. 1687 and 1954) and by the Institutional Animal Use and Care Committee of Mt. Sinai School of Medicine.

Nanoparticle synthesis. Pluronic-stabilized PPS nanoparticles with diameters of 25 and $100 \mathrm{~nm}$ were synthesized by inverse emulsion polymerization as described elsewhere ${ }^{8}$.

Antigen-conjugated nanoparticles. Antigen conjugation to PPS nanoparticles was accomplished by functionalizing Pluronic F-127 surface with the model protein antigen, ovalbumin. The conjugation scheme began with synthesis of Pluronic divinylsulfone, to which ovalbumin was coupled through a free thiol group on ovalbumin in a Michael-type addition reaction. Pluronic vinylsulfone conjugated to ovalbumin was then blended with conventional Pluronic and nanoparticles were synthesized as usual.

Fluorescence microlymphangiography. A constant-pressure infusion of fluorescently labeled nanoparticles was performed as previously described ${ }^{19}$ in order to visualize the lymphatic capillary network in the tail skin of mice.

C3a detection. A C3a sandwich enzyme-linked immunosorbent assay (ELISA) was performed to measure complement activation in human serum after incubation with polyhydroxylated- or polymethoxylated nanoparticles.

CD4 T-cell proliferation. T cells from OT-II mice were isolated and labeled with 5-(6)-carboxyfluorescein diacetate succinimidyl diester (CFSE) and were then adoptively transferred into wild-type (WT) mice. After 2 d, control, antigen or nanoparticle treatments were injected into mice. At $5 \mathrm{~d}$, lymph node cells were isolated and the dilution of CFSE was measured by flow cytometry.

CD8 T-cell memory. Mice received various intradermal injections of antigen and nanoparticle formulations into dorsal foot skin, with a booster at $7 \mathrm{~d}$ and at $21 \mathrm{~d}$. T cells were isolated from the draining lymph node, exposed to an ovalbumin-specific major histocompatibility-I peptide, and IFN- $\gamma$ production was measured through ELISPOT.

Ovalbumin antibody titers. A direct ELISA against ovalbumin was performed to detect the presence of anti-ovalbumin IgG in mouse serum.

Additional methods. Detailed methods are available in the Supplementary Methods online.

Note: Supplementary information is available on the Nature Biotechnology website.

\section{ACKNOWLEDGMENTS}

We thank M. Pasquier, V. Borel, V. Garea for valuable technical assistance; J.M. Rutkowski for scientific discussions; J.B. Dixon for MATLAB programming. Project funded by the Competence Centre for Materials Science and Technology (CCMX) of the ETH-Board, Switzerland (to M.A.S. and J.A.H.).

\section{AUTHOR CONTRIBUTIONS}

S.T.R. designed and performed the research, analyzed the data and wrote the manuscript; A.J.v.d.V. synthesized materials and analyzed synthetic data; E.S. developed methods on T-cell activation and analyzed the data; V.A. performed the research on T-cell adoptive transfer, analyzed the data and provided useful discussion on interpretation; G.J.R. analyzed the data and provided useful discussion on interpretation; C.P.O. synthesized materials and analyzed synthetic data; L.K.L. developed methods on T-cell activation and antibody titer measurement; M.A.S. designed the research, analyzed the data and wrote the manuscript; J.A.H. designed the research, analyzed the data and wrote the manuscript.

\section{COMPETING INTERESTS STATEMENT}

The authors declare competing financial interests: details accompany the full-text HTML version of the paper at http://www.nature.com/naturebiotechnology/.

Published online at http://www.nature.com/naturebiotechnology/

Reprints and permissions information is available online at http://npg.nature.com/ reprintsandpermissions

1. Bonifaz, L.C. et al. In vivo targeting of antigens to maturing dendritic cells via the DEC-205 receptor improves T cell vaccination. J. Exp. Med. 199, 815-824 (2004).

2. Dudziak, D. et al. Differential antigen processing by dendritic cell subsets in vivo. Science 315, 107-111 (2007).

3. Kwon, Y.J., James, E., Shastri, N. \& Frechet, J.M. In vivo targeting of dendritic cells for activation of cellular immunity using vaccine carriers based on $\mathrm{pH}$-responsive microparticles. Proc. Natl. Acad. Sci. USA 102, 18264-18268 (2005).

4. Trumpfheller, C. et al. Intensified and protective CD4+ T cell immunity in mice with anti-dendritic cell HIV gag fusion antibody vaccine. J. Exp. Med. 203, 607-617 (2006).

5. Wang, C. et al. Molecularly engineered poly(ortho ester) microspheres for enhanced delivery of DNA vaccines. Nat. Mater. 3, 190-196 (2004).

6. O'Hagan, D.T. \& Valiante, N.M. Recent advances in the discovery and delivery of vaccine adjuvants. Nat. Rev. Drug Discov. 2, 727-735 (2003).

7. Ulevitch, R.J. Therapeutics targeting the innate immune system. Nat. Rev. Immunol. 4 512-520 (2004)

8. Rehor, A., Hubbell, J.A. \& Tirelli, N. Oxidation-sensitive polymeric nanoparticles. Langmuir 21, 411-417 (2005).

9. Wilson, N.S. et al. Most lymphoid organ dendritic cell types are phenotypically and functionally immature. Blood 102, 2187-2194 (2003).

10. Reddy, S.T., Swartz, M.A. \& Hubbell, J.A. Targeting dendritic cells with biomaterials: developing the next generation of vaccines. Trends Immunol. 27, 573-579 (2006).

11. Randolph, G.J., Angeli, V. \& Swartz, M.A. Dendritic-cell trafficking to lymph nodes through lymphatic vessels. Nat. Rev. Immunol. 5, 617-628 (2005).

12. Pack, D.W. Timing is everything. Nat. Mater. 3, 133-134 (2004).

13. Little, S.R. et al. Poly-beta amino ester-containing microparticles enhance the activity of nonviral genetic vaccines. Proc. Natl. Acad. Sci. USA 101, 9534-9539 (2004).

14. Storni, T. et al. Nonmethylated CG motifs packaged into virus-like particles induce protective cytotoxic $\mathrm{T}$ cell responses in the absence of systemic side effects. J. Immunol. 172, 1777-1785 (2004).

15. Fifis, T. et al. Size-dependent immunogenicity: therapeutic and protective properties of nano-vaccines against tumors. J. Immunol. 173, 3148-3154 (2004).

16. van Broekhoven, C.L., Parish, C.R., Demangel, C., Britton, W.J. \& Altin, J.G. Targeting dendritic cells with antigen-containing liposomes: a highly effective procedure for induction of antitumor immunity and for tumor immunotherapy. Cancer Res. 64 4357-4365 (2004). 
17. Swartz, M.A. The physiology of the lymphatic system. Adv. Drug Deliv. Rev. 50, 3-20 (2001)

18. Swartz, M.A. \& Fleury, M.E. Interstitial flow and its effects in soft tissues. Annu. Rev. Biomed. Eng. 9, 229-256 (2007).

19. Reddy, S.T., Rehor, A., Schmoekel, H.G., Hubbell, J.A. \& Swartz, M.A. In vivo targeting of dendritic cells in lymph nodes with poly(propylene sulfide) nanoparticles. J. Control. Release 112, 26-34 (2006).

20. Nishioka, Y. \& Yoshino, H. Lymphatic targeting with nanoparticulate system. Adv. Drug Deliv. Rev. 47, 55-64 (2001).

21. Reddy, S.T., Berk, D.A., Jain, R.K. \& Swartz, M.A. A sensitive in vivo model for quantifying interstitial convective transport of injected macromolecules and nanoparticles. J. Appl. Physiol. 101, 1162-1169 (2006). 응 22. Boscardin, S.B. et al. Antigen targeting to dendritic cells elicits long-lived T cell help for

23. Carroll, M.C. The complement system in regulation of adaptive immunity. Nat. Immunol. 5, 981-986 (2004).
24. Dempsey, P.W., Allison, M.E., Akkaraju, S., Goodnow, C.C. \& Fearon, D.T. C3d of complement as a molecular adjuvant: bridging innate and acquired immunity. Science 271, 348-350 (1996).

25. Kemper, C. \& Atkinson, J.P. T-cell regulation: with complements from innate immunity. Nat. Rev. Immunol. 7, 9-18 (2007).

26. Kopf, M., Abel, B., Gallimore, A., Carroll, M. \& Bachmann, M.F. Complement component C3 promotes T-cell priming and lung migration to control acute influenza virus infection. Nat. Med. 8, 373-378 (2002).

27. Gadjeva, M. et al. The covalent binding reaction of complement component C3. J. Immunol. 161, 985-990 (1998).

28. Tang, L., Liu, L. \& Elwing, H.B. Complement activation and inflammation triggered by model biomaterial surfaces. J. Biomed Mater. Res. 41, 333-340 (1998).

29. Nilsson, B., Ekdahl, K.N., Mollnes, T.E. \& Lambris, J.D. The role of complement in biomaterial-induced inflammation. Mol. Immunol. 44, 82-94 (2007).

30. Kidane, A. \& Park, K. Complement activation by PEO-grafted glass surfaces. J. Biomed Mater. Res. 48, 640-647 (1999). 